\title{
1 Effects of transient blur and VDT screen luminance changes on eyeblink rate
}

2 ABSTRACT

3 Purpose: A study was designed to evaluate the efficacy of three different strategies aiming at increasing spontaneous eyeblink rate (SEBR) during computer use.

Methods: A total of 12 subjects ( 5 female) with a mean age of 28.7 years were instructed to read a text presented on a computer display terminal during 15 minutes. Four reading sessions (reference and three "blinking events" [BE]) were programmed in which SEBR was digitally recorded. "Blinking events" were based on either a slight distortion of the text characters or on the presentation of a white screen instead of the text, with or without accompanying blinking instructions. All BE had a duration of $20 \mathrm{~ms}$ and occurred every $15 \mathrm{~s}$. Participants graded the intrusiveness of each BE configuration, and the number of lines participants read in each session was recorded.

Results: Data from 11 subjects was analysed. A statistically significant difference in SEBR was encountered between the experimental configuration consisting on a white screen plus blinking instructions ( 7.8 blinks $/ \mathrm{min}$ ) and both reference $(5.2$ blinks $/ \mathrm{min} ; \mathrm{p}=0.049$ ) and white screen without blinking instructions ( 4.8 blinks $/ \mathrm{min} ; \mathrm{p}=0.038$ ). All three $\mathrm{BE}$ had superior levels of intrusiveness than reference conditions, although the performance of participants (line count) was not compromised.

Conclusions: The joint contribution of white screen and blinking instructions has been shown to result in a short term improvement in blinking rate in the present sample of non-dry eye computer users. Further work is necessary to improve the acceptance of any BE aiming at influencing SEBR.

\section{KEY WORDS}


2 Dry eye is one of the most frequently reported ocular complaint of visual display terminal

3 (VDT) users, with a range of 26.9 to $56 \%$ in prevalence in various studies [1,2]. This condition

4 has been found to arise from the joint contribution of an increased palpebral aperture (mainly

5 for desktop computer users) and altered blinking patterns (rate, amplitude and interblink

6 regularity) $[3,4]$. In addition, several factors may modulate the symptomatology described by

7 patients, such as distance from the screen, screen type, background luminance, rate of visual

8 information presented on the screen, screen refresh rate, glare sources, and room humidity,

9 temperature and ventilation [5-12].

Blinking has been described to occur voluntarily, reflexively or spontaneously [13]. Reflex blinks are evoked by external stimuli (including auditory, flashes of light, and mechanical stimulation of the ocular surface or surrounding structures), or by the ocular dryness that accompanies the destabilization and rupture of the tear film [14]. Conversely, spontaneous blinks are linked to cognitive processes, that is, seem to be regulated by a central "pacemaker" which is highly sensitive to the cognitive workload of the undergoing visual task $[15,16]$. Indeed, spontaneous eyeblink rate (SEBR) values have been found to range from 4.5 blinks/min while reading, to 17 blinks/min in silent primary gaze position (looking straight ahead and in silence at a distant target), further increasing to 26 blinks/min while subjects were engaged in conversation [17]. Besides, a significant difference in eyeblink patterns between tasks has been reported, with eyeblinks during conversation displaying a highly irregular behavior in which most eyeblinks were clustered into short sequences [15].

The influence of cognitive and attentional factors on blinking parameters is particularly relevant. Thus, while overall reductions in SEBR of up to $42 \%$ have been reported in subjects instructed to perform an attentive computer task [18,19], other authors have compared different tasks, observing the influence of the actual cognitive demands of the task at hand on 
1 SEBR. Skotte and coworkers noted a change in SEBR from 16 blinks/min to 5 blinks/min when

2 comparing passive (watching of a film) to active tasks (requiring subjects to connect a sequence of small dots on the screen) [20]. Similarly, Himebaugh et al, evaluated SEBR, blink amplitude and tear breakup while participants conducted a series of VDT tasks which had been previously classified as either low concentration tasks (looking at a blank computer screen or watching a movie) or high concentration tasks (playing a computer game or viewing a series of rapidly changing letters) [21]. The authors encountered reduced blinking rates during high concentration activities and documented a higher fluctuation in SEBR during the computer game trial (the associated blink amplitude was of about 50\%), an absence of full blinks and a consistent inferior area of tear breakup.

Previous attempts at improving SEBR during VDT tasks have met with diverse degrees of success. These attempts have included, among others, the use of antireflection films on computer displays, sudden changes in background lighting conditions and auditory stimuli [2224], with authors noting that the most effective strategies were also those being reported by participants as causing the highest levels of interference with the task they were performing [24]. It was therefore the aim of the present study to explore subtle, less-intrusive strategies which may lead to an increase in SEBR during computer work. Accordingly, computer programming was employed to design three different experimental conditions, described as "blinking events" (BE), and SEBR was monitored while subjects were instructed to read a text on a computer screen. The level of intrusiveness of each BE was determined both objectively

21 (line counting) and subjectively (vertical visual analogue scale) and the relationship between the level of intrusiveness and the other variables was investigated. 
1

2 Participants

3 A total of 12 subjects ( 5 female), with mean age of $28.7 \pm 5.8$ years (mean \pm SD), range from 22

4 to 43 years, were recruited for this study. All subjects had good ocular health, distance and

5 near binocular visual acuity of 1 (decimal) or better with habitual correction, TBUT score $>10 \mathrm{~s}$

6 and OSDI score $<15[25,26]$. Subjects with binocular vision problems, amblyopia, oculomotor

7 anomalies and eyelid position (such as ptosis) or movement abnormalities were excluded from

8 the study. Contact lens wear was not an exclusion criterion, per se, although participants were

9 asked to use their spectacles instead of their contact lenses on the days they took part in the

10 study.

11 All participants provided written informed consent after the nature of the study was explained

Blinking events

Four experimental configurations were implemented, including reference (BL) and three different "blinking events" (BE). Microsoft Visual Basic 10 programming language for Windows (Microsoft Ireland Operations Ltd, Dublin, Ireland) was employed to introduce small modifications on how the text appeared on the screen. For BE1 a white rectangle was briefly superimposed on the text. The BE2 experimental configuration consisted in a slight nonquantifiable distortion (doubling plus blurring) of the text characters (Figure 1). Finally, BE3 was identical to BE1, with the addition that participants were explicitly encouraged to blink in 
1

2

synchronicity with the presentation of the white screen. BE3 always took place at the last reading session, as it was considered that, by providing specific blinking instructions, the purpose of the study was partially revealed. All BE had a duration of $20 \mathrm{~ms}$ and were programmed to occur every $15 \mathrm{~s}$. For BL no "blinking event" was introduced while participants read the text presented on a computer screen.

\section{Procedure}

Four reading sessions were scheduled (on consecutive days) in which, under the same, controlled conditions, SEBR was digitally recorded. Reference, BE1 and BE2 sessions took place, in random order, on the first three reading sessions. Conversely, BE3 always took place at the last reading session.

At each session participants were instructed to silently read a text presented on a computer display for 15 minutes. Text, which consisted in a collection of easy-reading short stories by famous authors presented in Arial 12, was displayed on a 20 inch liquid crystal display (TFTLCD) computer screen set to a resolution of 1280 per 1024 pixels, 32 bit colour configuration, contrast ratio $700: 1$ and $75 \mathrm{~Hz}$ refresh rate. Participants viewed the display from a distance of $50 \mathrm{~cm}$ with head fixed in a chin and forehead rest, which height could be adjusted, as well as that of the chair and computer desk, to ensure subject comfort and to align the centre of the screen at the level of the participant's eyes. The inclination angle of the screen was of 100 degrees from the plane of the computer desk. Display luminance was set at approximately 15 $\mathrm{cd} / \mathrm{m}^{2}$. Participants scrolled down the text with the aid of the central wheel of a mouse.

A screen mounted Logitech HD Webcam C310 (Logitech España BCN, S.L., Barcelona, Spain) was employed to record the eyes of the participants, whereupon images were visually examined to determine SEBR using the Logitech Webcam software 2.51.828.0 for Windows. All 
1

2

3

4

5

6

eyeblinks were counted, although eyelid twitching or small downward movements of the upper eyelid, not covering more than one third of the pupil, were ignored. One eye at random was selected to determine SEBR.

Room temperature and humidity were maintained at $20^{\circ} \mathrm{C}\left( \pm 2^{\circ} \mathrm{C}\right)$ and $40 \%( \pm 10 \%)$ throughout the study, respectively. Background illumination was set at approximately 400 lux, and provided by diffuse lighting to avoid unwanted screen reflections. All reading sessions took place between $10.00 \mathrm{~h}$. and $14.00 \mathrm{~h}$.

Subjective level of intrusiveness (SLI) for each BE configuration was graded from 0 "It does not get in the way of my reading" to 100 "I am not able to read at all" on a $100 \mathrm{~mm}$ vertical visual analogue scale [27]. In addition, the number of text lines each participant was able to read in each reading session was also recorded. Lines were recorded by highlighting the text that each participant was able to read during each reading session and using the Word command "Word Count" to generate a report listing the number of lines in that fragment of text.

\section{Data analysis}

Although all variables (SEBR, SLI and text line count) were quantitative and continuous in nature, they were submitted to the Kolmogorov-Smirnov test of normality prior to any further statistical analysis, which revealed many instances of non-normality $(p<0.05)$. Therefore, data presentation is based on median and range (maximum - minimum) values, although mean and standard deviation (SD) is also provided for comparison purposes. The non-parametric Friedman's test for repeated measures and the Wilcoxon signed-ranks test were used to evaluate overall and pair-wise differences in SEBR, SLI and text line count between BL, BE1, BE2 and BE3, respectively. In addition, the Spearman's rho correlation test was employed to 
1 determine the relationship between the variables under study. A p-value of 0.05 or less was

2 considered to denote statistical significance throughout the study. 


\section{RESULTS}

All participants successfully completed the four reading sessions. However, a preliminary data review exposed one participant with a reference SEBR of 29 blinks/min. The SEBR scores for this participant were consequently excluded from any further statistical analysis. Therefore, the final number of participants of the study was 11.

Table 1 and Table 2 present a summary (in median and range, as well as mean and SD) of SEBR, SLI and line count, for reference and for each of the three BE experimental configurations. Figures 2, $\mathbf{3}$ and $\mathbf{4}$ display box plot representations of SEBR, SLI and line count for each reading session, respectively. A Friedman's test for repeated measures revealed a statistically significant difference in SEBR between the four reading sessions $\left(\chi^{2}=7.909 ; p=\right.$ 0.048) which, when submitted to a pair-wise analysis with the Wilcoxon signed-ranks test, disclosed statistically significant differences between several experimental configurations. The same statistical approach revealed a statistically significant difference in SLI between reading sessions $\left(\chi^{2}=24.321 ; p<0.001\right)$. All three $B E$ experimental configurations resulted in an increase in SLI when compared with BL scores, with text character distortion (BE2) graded as the most intrusive of all BE. Finally, no statistically significant differences were encountered in the number of lines of text participants were able to read during the four sessions.

Upon examining data as classified by reading session, statistically significant strong correlations in SEBR scores were found between BL and BE1 (rho $=0.683 ; p=0.042$ ) and BE2 (rho $=0.733 ; p=0.025$ ), as well as between BE1 and BE2 (rho $=0.698 ; p=0.040$ ). Not unexpectedly, as reading speed may be considered a participant specific skill, strong correlations were also found between all line counts (all rho $>0.8$; all $p<0.05$ ). Interestingly, a strong, statistically significant negative correlation was encountered between the SLI score and line count of the BE3 experimental setting ( $r$ ho $=-0.816 ; p=0.007$ ), which was not evidenced between any other pairs of SLI scores and line counts. 


\section{DISCUSSION}

The purpose of the present study was to evaluate different strategies that, while aiming at improving SEBR in computer users, did not interfere with their performance, as graded both subjectively through a vertical visual analogue scale and objectively, by counting the lines of text participants were able to read in each of the 15 minutes sessions, which was considered an objective indication of the level of intrusiveness of each BE.

Overall, a median of 5.2 blinks/min was recorded in reference conditions, in which participants were instructed to silently read a text presented on a desktop computer screen. This SEBR is in agreement with previous studies assessing blinking in similar conditions (although in some of these studies viewing distance was not adjusted with a chin and forehead rest) [15, 17-21]. A statistically significant difference in SEBR was encountered between the four reading sessions, with pair-wise analysis revealing statistically significant differences between $B L$ and $B E 3$ and between $\mathrm{BE} 1$ and $\mathrm{BE} 3$. It must be noted that the present experimental configuration for all three $\mathrm{BE}$ consisted in a short $(20 \mathrm{~ms})$ presentation, with a repetition interval of $15 \mathrm{~s}$. These particular settings were selected after initial trials in which a low level of intrusiveness was prioritized over the possible efficacy of each BE in improving SEBR. It may be speculated whether, with a shorter repetition interval, the improvement in SEBR encountered in BE3 would have been more pronounced. Similarly, it would be very interesting to evaluate SEBR, SLI and line count (or another indicator of the performance of computer users) over a longer period of time, approximating actual working hours.

Previous researchers have evaluated different strategies aiming at improving SEBR. MiyakeKashima and co-workers described an improvement in aesthenopic symptoms and an increase in SEBR from $9.6 \pm 4.3$ to $14.3 \pm 9.2$ blinks/min when participants viewed a movie on their computer display terminal without and with an antireflection film interposed on the screen [22]. These authors suggested that unwanted reflections, which were associated with image 
1

2

3

4

5

6

7

8

degradation and loss of contrast, resulted in observers requiring higher levels of attention when viewing the movie, with a subsequent negative impact on SEBR. Doughty, on the other hand, showed a transient increase in SEBR (from $12.3 \pm 1.6$ to $21.6 \pm 4.6$ blinks/min) when the background was suddenly flooded with light [23]. Finally, Portello and co-workers instructed their participants to blink on hearing a tone (an audible beep with a repetition interval of $4 \mathrm{~s}$ ), recording an increase in SEBR from $11.29 \pm 1.67$ to $23.45 \pm 1.65$ blinks/min, when compared with reference conditions [24].

It may be observed that these studies follow two main strategic approaches to improve SEBR: they either elicit some type of reflex blinking or they aim at influencing the regulation of the central blinking "pacemaker", which has been shown to be highly sensitive to the cognitive demands of the undergoing task [18-21]. It may be assumed that strategies relying on a sustained contribution of reflex blinking might be graded by participants as more intrusive than those based on subtle modulations of the activity of the internal "pacemaker" [28]. This hypothesis would be in agreement with the high intrusiveness score awarded to the second experimental configuration, in which reflex blinking was elicited by a sudden distortion of the text characters. Indeed, the blinking stimulus behind BE2 may be considered as equivalent to the slight loss of retinal image quality resulting from tear film instability and breakup $[29,30]$ which, in addition to ocular surface dryness as detected by the trigeminal nerve terminals, may contribute in triggering reflex blinking.

It is interesting to note that the inclusion of a $20 \mathrm{~ms}$ white screen in front of the text did not lead to a significant increase in SEBR, if unaccompanied by explicit blinking instructions. This finding was unexpected, as the short presentation of a white screen was believed to be able to benefit from both strategic approaches described above. Thus, on the one hand, the sudden slight increase in display luminance was thought to be able to elicit reflex blinking. On the other hand, the regulation of the central "pacemaker" might also be influenced, as previous 
1 studies have reported spontaneous blinking to occur in synchronicity with explicit or implicit

2 attentional breaks in the continuous flow of visual information [31]. The encountered

3 differences between $\mathrm{BL}$ and $\mathrm{BE} 3$ and between $\mathrm{BE} 1$ and $\mathrm{BE} 3$, however, suggest a predominant

4 contribution of the second strategic approach, which may be assumed to profit from blinking

5 instructions.

6 These findings support the need to explore the possibility of eyeblink conditioning. In effect, it

7 has been shown that with repeated paired presentations of a tone-conditioning stimulus

8 preceding an airpuff unconditioned stimulus, a conditioned eyeblink develops, which precedes

9 the airpuff [32]. It may be speculated whether precise blinking instructions, when paired with a

10 non intrusive $\mathrm{BE}$, may lead to eyeblink conditioning resulting in a sustained improvement in

11 SEBR, even in the subsequent absence of BE.

12 In conclusion, the present findings suggest that SEBR may be altered by different strategic 13 approaches, and careful consideration of their level of intrusiveness is required to avoid 14 interfering with the performance of computer users, with further work being necessary to 15 improve the acceptance of any BE aiming at influencing SEBR. Albeit further studies are necessary to investigate the long term effectiveness of these strategies, as far as we know the

17 present results signify the first steps in the exploration of this very relevant and fertile area of research. 


\section{REFERENCES}

1. Uchino M, Schaumberg DA, Dogru M, Uchino Y, Fukagawa K, Shimmura S, Satoh T, Takebayashi T, Tsubota K. Prevalence of dry eye disease among Japanese visual display terminal users. Ophthalmology 2008; 15: 1982-8.

2. Hayes JR, Sheedy JE, Stelmack JA, Heaney CA. Computer use, symptoms, and quality of life. Optom Vis Sci 2007; 84: E739-45.

3. Tsubota K, Nakamori K. Effects of ocular surface area and blink rate on tear dynamics. Arch Ophthalmol 1995; 113: 155-8.

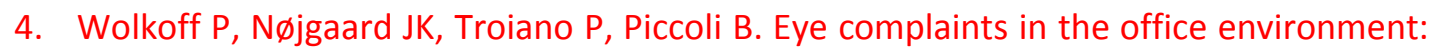
precorneal tear film integrity influenced by eye blinking efficiency. Occup Environ Med 2005; 62: 4-12.

5. Bhanderi DJ, Choudhary S, Doshi VG. A community-based study of asthenopia in computer operators. Indian J Ophthalmol 2008; 56: 51-5.

6. Lin YH, Chen CY, Lu SY, Lin YC. Visual fatigue during VDT work: effects of time-based and environment-based conditions. Displays 2008; 29: 487-92.

7. Hollands JG, Parker HA, McFadden S, Boothby R. LCD versus CRT displays: a comparison of visual search performance for colored symbols. Hum Factors 2002; 44: 210-21.

8. Sheedy J, Smith R, Hayes J. Visual effects of the luminance surrounding a computer display. Ergonomics 2005; 48: 1114-28.

9. Mendell MJ, Fisk WJ, Petersen MR, Hines CJ, Dong M, Faulkner D, Deddens JA, Ruder AM, Sullivan D, Boeniger MF. Indoor particles and symptoms among office workers: results from a double-blind cross-over study. Epidemiology 2002; 13: 296-304.

10. Shieh KK, Lai YK. Effects of ambient illumination, luminance contrast, and stimulus type on subjective preference of VDT target and background color combinations. Percept Mot Skills 2008; 107: 236-52.

11. Cardona G, García C, Serés C, Vilaseca M, Gispets J. Blink rate, blink amplitude, and tear film integrity during dynamic visual display terminal tasks. Curr Eye Res 2011; 36: 190-7.

12. Jaschinski W, Bonacker M, Alshuth E. Accommodation, convergence, pupil diameter and eye blinks at a CRT display flickering near fusion limit. Ergonomics 1996; 39: 15264.

13. Manning KA, Evinger C. Different forms of blinks and their two-stage control. Exp Brain Res 1986; 64: 579-88. 
14. Holly FJ. Formation and rupture of the tear film. Exp Eye Res 1973; 15: 515-25.

15. Doughty MJ. Consideration of three types of spontaneous eyeblink activity in normal humans: during reading and video display terminal use, in primary gaze, and while in conversation. Optom Vis Sci 2001; 78: 712-25.

16. Veltman JA, Gaillard AW. Physiological workload reactions to increasing levels of task difficulty. Ergonomics. 1998; 41: 656-669.

17. Bentivoglio AR, Bressman SB, Cassetta E, Carretta D, Tonali P, Albanese A. Analysis of blink rate patterns in normal subjects. Mov Disord 1997; 12: 1028-34.

18. Acosta MC, Gallar J, Belmonte C. The influence of eye solutions on blinking and ocular comfort at rest and during work at video display terminals. Exp Eye Res 1999; 68: 6639.

19. Freudenthaler N, Neuf H, Kadner G, Schlote T. Characteristics of spontaneous eyeblink activity during video display terminal use in healthy volunteers. Graefe's Arch Clin Exp Ophthalmol 2003; 241: 914-20.

20. Skotte JH, Nøjgaard JK, Jørgensen LV, Christensen KB, Sjøgaard G. Eye blink frequency during different computer tasks quantified by electrooculography. Eur J Appl Physiol 2007; 99: 113-9.

21. Himebaugh NL, Begley CG, Bradley A, Wilkinson JA. Blinking and tear break-up during four visual tasks. Optom Vis Sci 2009; 86: E106-14.

22. Miyake-Kashima M, Dogru M, Nojima T, Murase M, Matsumoto Y, Tsubota K. The effect of antireflection film use on blink rate and asthenopic symptoms during visual display terminal work. Cornea 2005; 24: 567-70.

23. Doughty MJ. Effects of background lighting and retinal illuminance on spontaneous eyeblink activity of human subjects in primary eye gaze. Eye Contact Lens 2013; 39: 138-46.

24. Portello JK, Rosenfield M, Chu CA. Blink rate, incomplete blinks and computer vision syndrome. Optom Vis Sci 2013; 90: 482-7.

25. Lemp MA. Report of the National Eye Institute/Industry Workshop on Clinical Trials in Dry Eye. CLAO J 1995; 23: 221-32.

26. Schiffman RM, Christianson MD, Jacobsen G, Hirsch JD, Reis BL. Reliability and validity of the Ocular Surface Disease Index. Arch Ophthalmol 2000; 118: 615-21.

27. Dixon JS, Bird HA. Reproducibility along a $10 \mathrm{~cm}$ vertical visual analogue scale. Ann Rheum Dis 1981; 40: 87-9. 
28. Doughty MJ, Naase T, Button NF. Frequent spontaneous eyeblink activity associated with reduced conjunctival surface (trigeminal nerve) tactile sensitivity. Graefes Arch Clin Exp Ophthalmol 2009; 247: 939-46.

29. Himebaugh NL, Nam J, Bradley A, Liu H, Thibos LN, Begley CG. Scale and spatial distribution of aberrations associated with tear breakup. Optom Vis Sci 2012; 89: 1590600.

30. Liu H, Thibos LN, Begley CG, Bradley A. Measurement of the time course of optical quality and visual deterioration during breakup. Invest Ophthalmol Vis Sci 2010; 51: 3318-26.

31. Nakano T, Yamamoto $\mathrm{Y}$, Kitajo K, Takahashi T, Kitazawa S. Synchronization of spontaneous eyeblinks while viewing video stories. Proc R Soc B 2009; 276: 3635-44.

32. Parker KL, Andreasen NC, Liu D, Freeman JH, Ponto LL, O'Leary DS. Eyeblink conditioning in healthy adults: a positron emission tomography study. Cerebellum 2012; 11: 946-56. 
2

3 Table 1: Spontaneous eyeblink rate (SEBR, in blinks/minute) for reference (BL) and for each of 4 the three "blinking events" (BE1: white rectangle; BE2: character distortion; BE3: white 5 rectangle + blinking instructions). All results are shown as mean, standard deviation (SD), 6 median and range. Any $p<0.05$, in bold, denotes statistical significance.

7

\begin{tabular}{cccc}
\hline & SEBR & $\begin{array}{c}\text { Overall Analysis } \\
\left(\mathbf{x}^{2}, \mathbf{p}\right)\end{array}$ \\
\hline BL & Mean & 5.6 & \\
& SD & 1.6 & \\
& Median & 5.2 & \\
& Range & $(1.7-8.6)$ & \\
BE1 & Mean & 6.1 & \\
& SD & 2.2 & \\
& Median & 4.8 & \\
& Range & $(2.5-9.6)$ & \\
& Mean & 7.909 & \\
BE2 & SD & 6.0 & \\
& Median & 6.5 & \\
& Range & $(2.5-22.8)$ & \\
& & & \\
& Mean & 11.0 & \\
BE3 & SD & 7.5 & \\
& Median & 7.8 & \\
& Range & $(3.9-24.0)$ & \\
\hline
\end{tabular}

8

9 Statistically significant pair-wise differences in SEBR were found between BL and BE3 ( $Z=-$ 10 1.963; $p=0.049)$ and between BE1 and BE3 $(Z=-2.073 ; p=0.038)$ 
1 Table 2: Subjective level of intrusiveness (SLI, $\mathrm{cm}$ ) and line count for reference $(\mathrm{BL})$ and for 2 each of the three "blinking events" (BE1: white rectangle; BE2: character distortion; BE3: white 3 rectangle + blinking instructions). All results are shown as mean, standard deviation (SD), 4 median and range. Any $p<0.05$, in bold, denotes statistical significance.

\begin{tabular}{|c|c|c|c|c|c|}
\hline & & SLI & $\begin{array}{c}\text { Overall } \\
\text { Analysis }\left(\chi^{2}, p\right)\end{array}$ & Line Count & $\begin{array}{l}\text { Overall Analysis } \\
\left(\chi^{2}, p\right)\end{array}$ \\
\hline \multirow{4}{*}{$\mathrm{BL}$} & Mean & 1.7 & & 180.6 & \multirow{16}{*}{$\begin{array}{c}5.667 \\
(0.129)\end{array}$} \\
\hline & SD & 0.5 & & 58.3 & \\
\hline & Median & 2 & & 167 & \\
\hline & Range & $(1-4)$ & & $(122-313)$ & \\
\hline \multirow{4}{*}{ BE1 } & Mean & 3.2 & & 185.2 & \\
\hline & SD & 1.0 & & 37.1 & \\
\hline & Median & 3 & & 169 & \\
\hline & Range & $(2-7)$ & 24.321 & $(147-238)$ & \\
\hline \multirow{4}{*}{ BE2 } & Mean & 66 & $(<0.001)$ & 165.4 & \\
\hline & SD & 1.7 & & 31.4 & \\
\hline & Median & 7 & & 162 & \\
\hline & Range & $4-9)$ & & $(130-234)$ & \\
\hline \multirow{4}{*}{ BE3 } & Mean & 4.4 & & 181.3 & \\
\hline & SD & 1.9 & & 33.3 & \\
\hline & Median & 4 & & 186 & \\
\hline & Range & $(2-9)$ & & $(140-247)$ & \\
\hline
\end{tabular}

6

7 Statistically significant pair-wise differences in SLI were found between BL and all BE (all $p<$ $80.05)$ as well as between BE2 and both BE1 $(Z=-2.675 ; p=0.007)$ and BE3 $(Z=-2.388 ; p=$ 9 0.017) 
FIGURES

2

3 Figure 1: Screen capture of the second "blinking event" (BE2), consisting in a slight distortion

4 of the text characters.

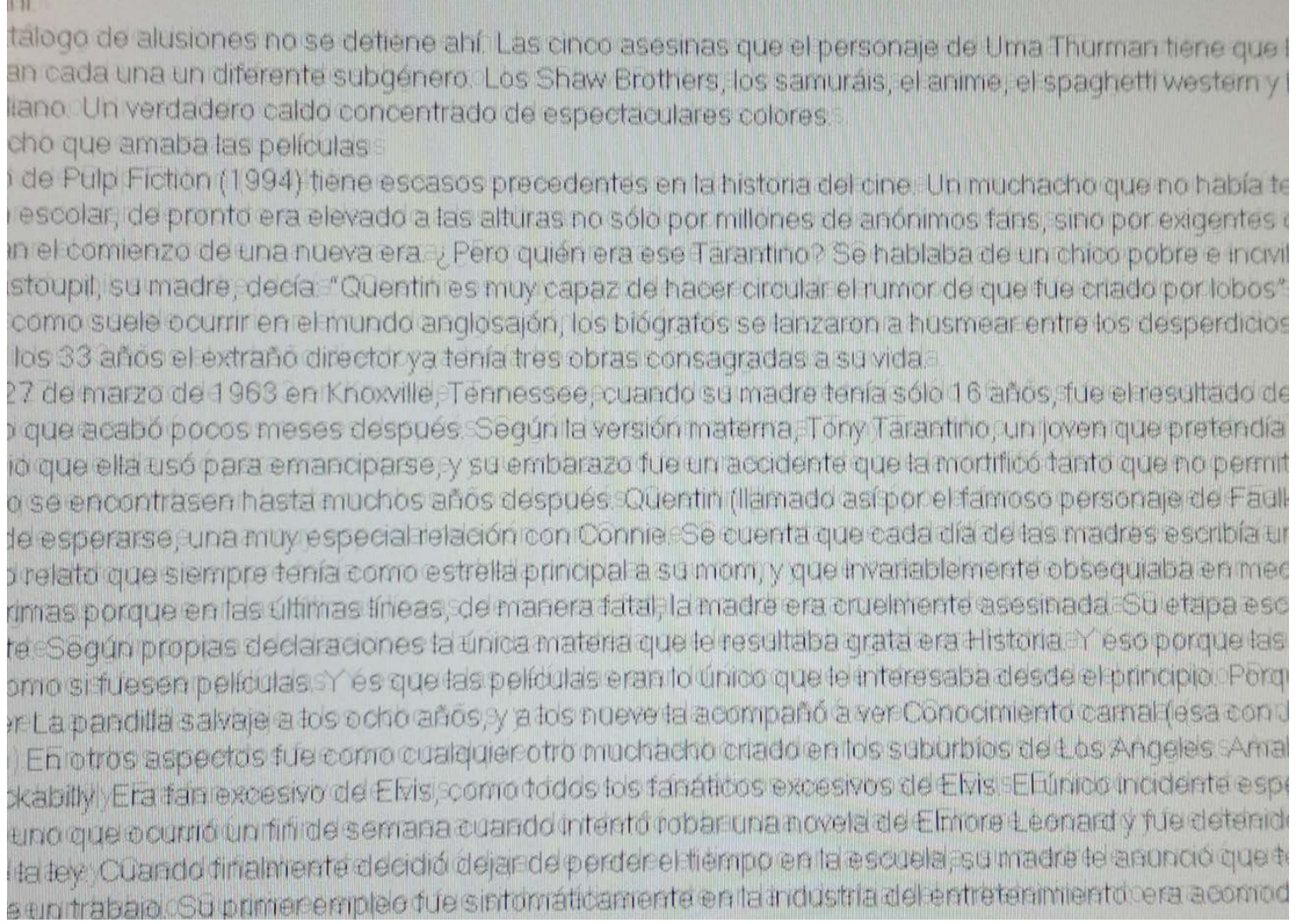


1 Figure 2: Box plot representation of spontaneous eyeblink rate (SEBR, in blinks/minute) for 2 each experimental configuration (reference: BL; white rectangle: BE1; character distortion: 3 BE2; white rectangle + blinking instructions: BE3).

4

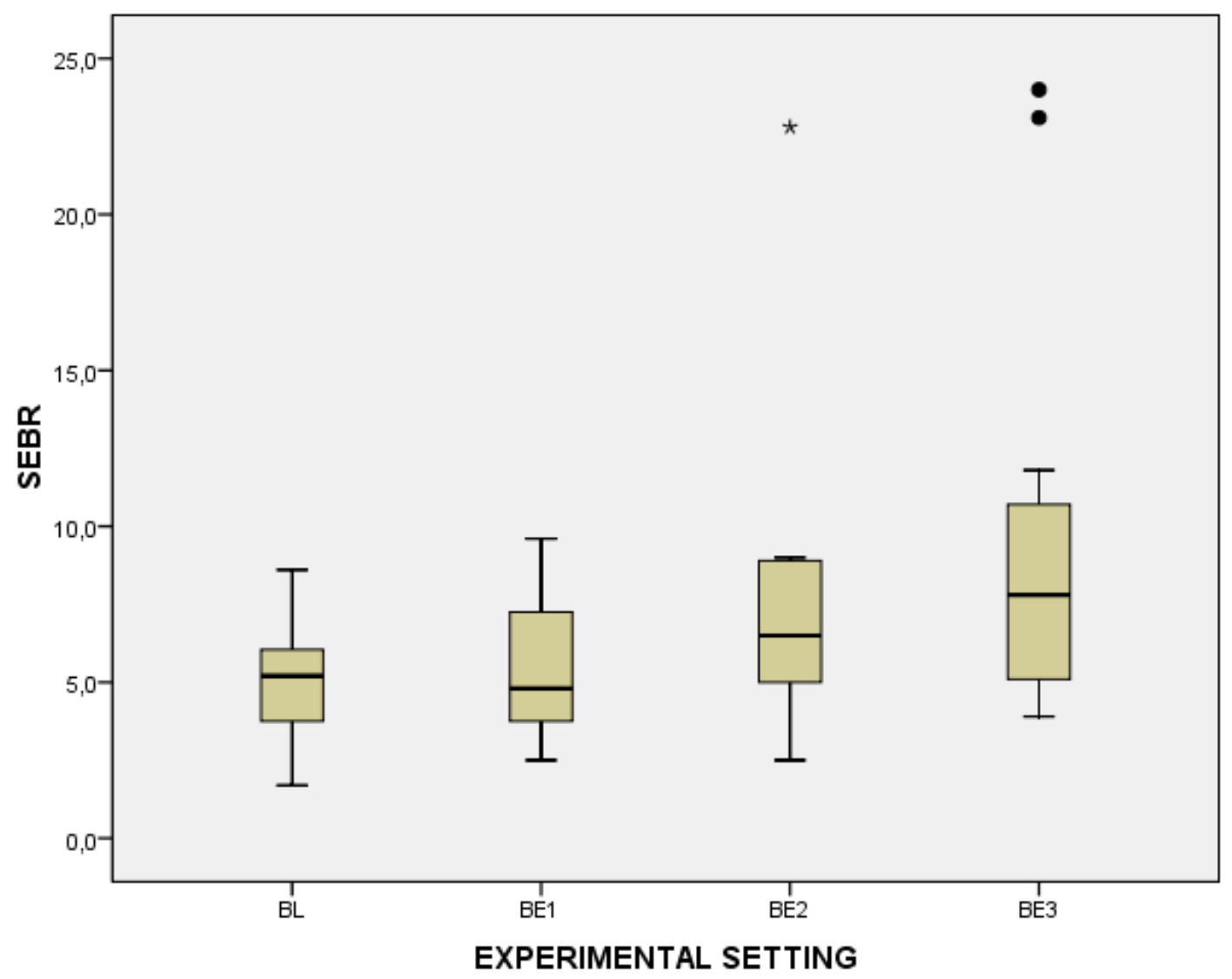

5

6 
1 Figure 3: Box plot representation of spontaneous subjective level of intrusiveness, as 2 measured on a vertical visual analogue scale $(10 \mathrm{~cm})$ for each experimental configuration 3 (reference: BL; white rectangle: BE1; character distortion: BE2; white rectangle + blinking 4 instructions: BE3).

5

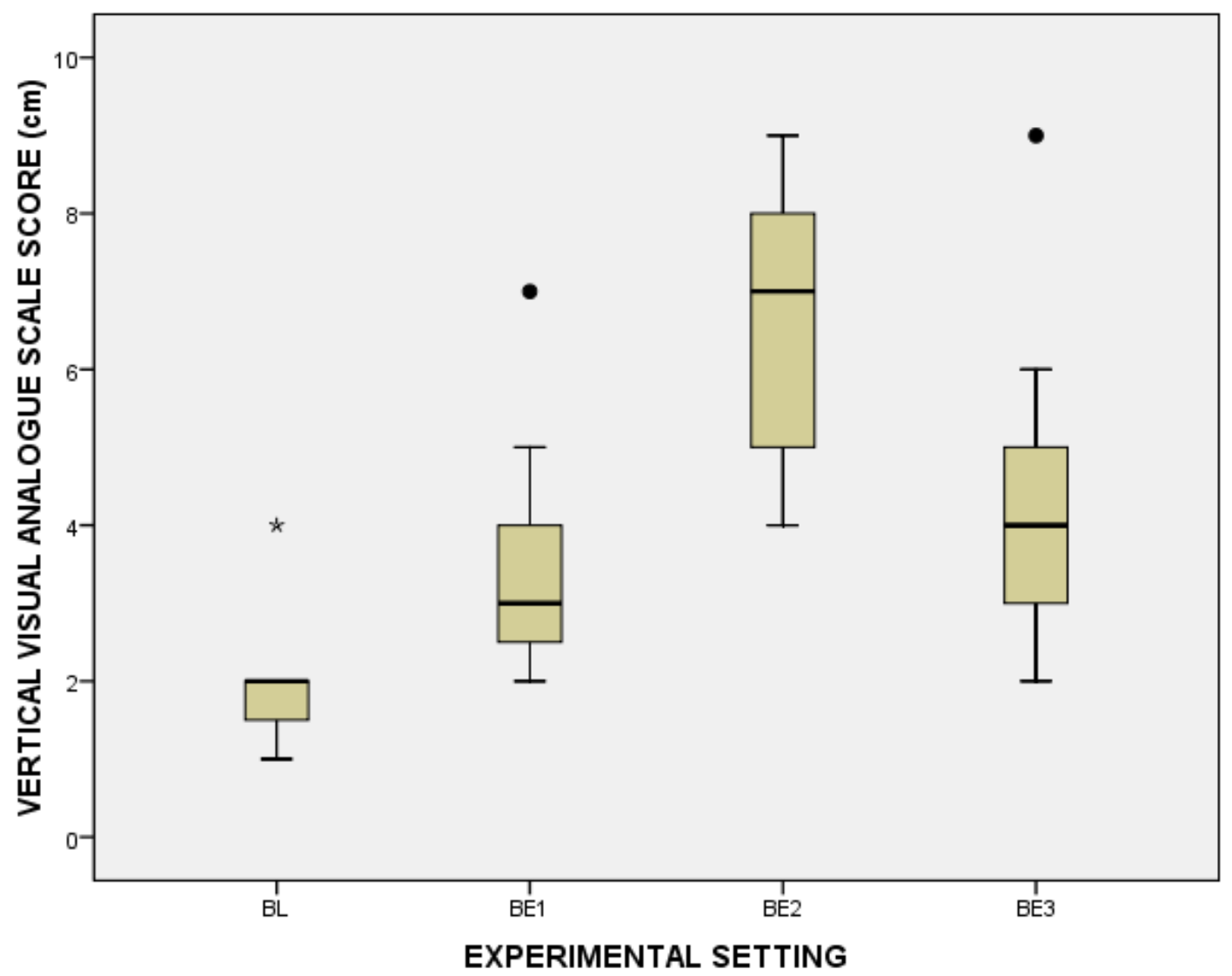

6 
1 Figure 4: Box plot representation of lines of text participants were able to read for each 2 experimental configuration (reference: BL; white rectangle: BE1; character distortion: BE2; 3 white rectangle + blinking instructions: $\mathrm{BE} 3)$.

4

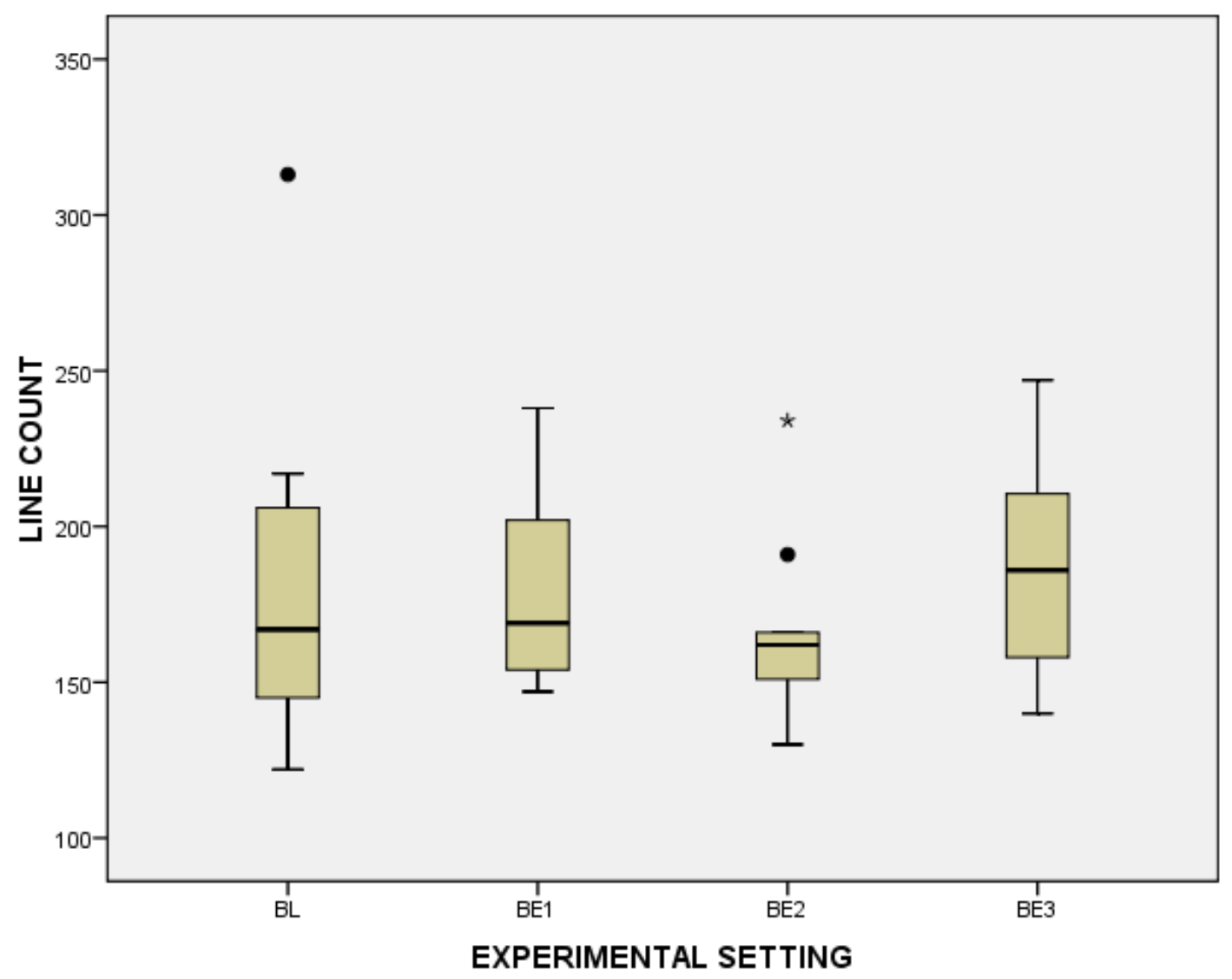

